\title{
Correction to: Proceedings of the 8th International Congress on Environmental Geotechnics Volume 2
}

Liangtong Zhan, Yunmin Chen, and Abdelmalek Bouazza

\section{Correction to:}

L. Zhan et al. (Eds.):

Proceedings of the 8th International Congress on Environmental Geotechnics Volume 2, ESE, https://doi.org/10.1007/978-981-13-2224-2

The original version of the book was inadvertently published without incorporating the author corrections. The book has been updated with the changes. 